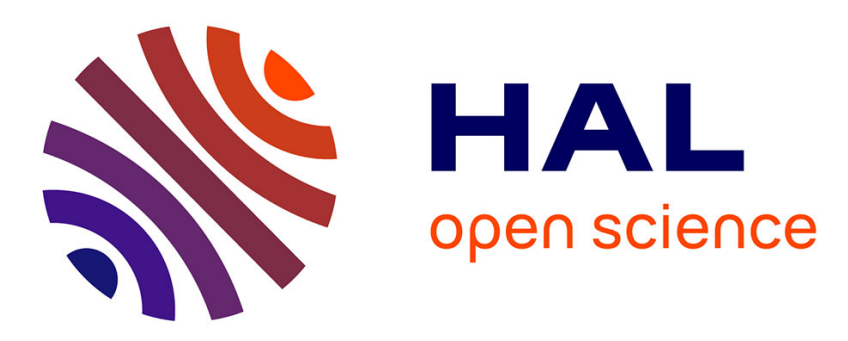

\title{
Facteurs psychosociaux des attitudes alimentaires déséquilibrées en contexte sportif: une revue de littérature
}

\author{
Stéphanie Scoffier-Mériaux, Fabienne d'Arripe-Longueville
}

\section{To cite this version:}

Stéphanie Scoffier-Mériaux, Fabienne d'Arripe-Longueville. Facteurs psychosociaux des attitudes alimentaires déséquilibrées en contexte sportif: une revue de littérature. Movement \& Sport Sciences Science \& Motricité, 2012, 76, pp.47 - 57. 10.1051/sm/2011123 . hal-02524784

\section{HAL Id: hal-02524784 \\ https://hal.univ-cotedazur.fr/hal-02524784}

Submitted on 6 Apr 2020

HAL is a multi-disciplinary open access archive for the deposit and dissemination of scientific research documents, whether they are published or not. The documents may come from teaching and research institutions in France or abroad, or from public or private research centers.
L'archive ouverte pluridisciplinaire HAL, est destinée au dépôt et à la diffusion de documents scientifiques de niveau recherche, publiés ou non, émanant des établissements d'enseignement et de recherche français ou étrangers, des laboratoires publics ou privés. 
Facteurs psychosociaux des attitudes alimentaires déséquilibrées en contexte sportif : une revue de littérature

Stéphanie Scoffier et Fabienne d'Arripe-Longueville

Université de Nice Sophia Antipolis, LAMHES, France

Re.cu le 9 septembre 2011 - Accepté Le le 29 juillet 2011 
1 Facteurs Psychosociaux des Désordres Alimentaires en Contexte Sportif : une Revue de sociales.

\section{Littérature}

\section{Résumé}

La prévalence des désordres alimentaires est particulièrement importante dans le contexte du sport de haut niveau, en particulier dans les sports d'endurance, les sports à catégorie de poids et les sports esthétiques. Dans cette revue de littérature, sont abordés la prévalence des désordres alimentaires en sport et les facteurs psychosociaux les plus à mêmes d'influencer le développement de ces désordres chez les sportifs engagés dans des sports à caractère esthétique. Si certains facteurs sont similaires à ceux identifiés dans l'étude des désordres alimentaires dans la population générale, d'autres apparaissent spécifiques au contexte social et aux normes d'accomplissement sportifs. Par exemple, l'émergence des désordres alimentaires dans les sports jugés sur des critères esthétiques sont fortement déterminés par la qualité de la relation sociale avec l'entraîneur, ou avec l'ami(e) en sport, et par l'habileté perçue. 
1

8 most apt to influence the development of these disorders in athletes of aesthetic sports.

9 Although some factors are similar to those identified in studies of the general population, 10 others appear to be quite specific to the social context and achievement norms of sport. For

11 example, the emergence of eating disorders in sports judged on aesthetic criteria is strongly determined by the quality of the athlete's relationship with the coach and by perceived ability.

13

14 Keywords: eating disorders, sport, norms, achievement motivation, self-esteem, social 15 influences. 
1

Facteurs Psychosociaux des Désordres Alimentaires en Contexte Sportif : une Revue de Littérature

\section{Introduction}

L'étude des désordres alimentaires chez les sportifs a connu depuis une quinzaine d'années un développement croissant (voir Petrie \& Greenleaf, 2007 ; Smolak, Murnen, \& Ruble, 2000 pour revues), ainsi qu'en témoignent plus de 200 articles référencés sur le sujet dans les bases de données PsychINFO et/ou Science Direct. Si certains des déterminants psychosociaux des désordres alimentaires semblent identifiables quel que soit le contexte (e.g., anxiété, influences des parents), d'autres apparaissent uniquement dans le contexte sportif. Dans cette revue de littérature, nous nous appuierons notamment sur les résultats de travaux récents étudiant, à partir de théories sociocognitives contemporaines, les désordres alimentaires de sportifs de haut niveau pratiquant des sports esthétiques.

La classification du DSM-IV est actuellement la référence pour définir les différents types de désordres alimentaires. Selon l'Institut National de la Santé et de la Recherche Médicale, les désordres alimentaires se définissent comme un ensemble de troubles caractérisés par des perturbations psychologiques conduisant à des désordres physiologiques de l'appétit et/ou de la consommation alimentaire. Ils sont associés à un trouble de l'image du corps et émergent majoritairement à l'adolescence. Fairburn et Harrison (2003) ont noté qu'environ la moitié des cas cliniques était classée dans les troubles alimentaires non spécifiés. Ainsi, dans cette revue de littérature, nous ne nous sommes pas cantonnés à l'anorexie sportive ou aux pathologies spécifiées dans le DSM-IV. Nous avons considéré les désordres alimentaires comme l'éventail des comportements malsains vis-à-vis de l'alimentation. Ils s'étendent des comportements pathologiques comme dans l'anorexie ou la boulimie, jusqu'aux comportements de consommation déséquilibrée qui aident à perdre du poids ou à maintenir un physique mince (Hobart \& Smucker, 2000). Ces désordres 
1 alimentaires sont acceptés comme un concept non clinique et proviennent de données auto-

2 rapportées par les individus.

Dans cet article, nous tenterons de montrer que les caractéristiques spécifiques du contexte sportif en termes de contexte social et de normes d'accomplissement, confèrent aux désordres alimentaires en sport un statut particulier. Afin de développer ce projet, nous examinerons dans un premier temps la prévalence ${ }^{1}$ des désordres alimentaires en sport, et notamment dans les disciplines à caractère esthétique. Dans un second temps, nous présenterons les principaux facteurs psychosociaux des désordres alimentaires chez ces sportifs. Enfin, nous évoquerons les perspectives de recherche prioritaires à développer sur ce thème.

Dans le contexte particulier du sport, Smolak et al. (2000) ont mis en évidence, au travers de leur méta-analyse reposant sur 34 études, que les désordres alimentaires sont plus fréquents chez les sportifs que chez les non-sportifs. En effet, un examen des études scientifiques récentes montre que $1 \%$ à $62 \%$ des athlètes présentent des désordres alimentaires (e.g., Sherman \& Thompson, 2009 ; Smolak et al., 2000; Sundgot-Borgen, 1994; SundgotBorgen \& Torstveit, 2004). Certains sports, pour lesquels la minceur est censée conférer des avantages compétitifs, seraient particulièrement « à risque » (Beals \& Manore, 2000 ; Sherman \& Thompson, 2009). Tel est le cas, (a) des sports où un faible poids est considéré comme pouvant contribuer à la vitesse et à l'efficience du mouvement (e.g., marathon, courses d'endurance); (b) des sports à catégorie de poids (e.g., sports de combat); et (c) des sports jugés sur des critères esthétiques (e.g., gymnastique, patinage artistique) qui nécessitent une morphologie spécifique (Sherman \& Thompson, 2009 ; Smolak et al., 2000;

\footnotetext{
1 Rapport du nombre de cas d'un trouble morbide à l'effectif total d'une population, sans distinction entre les cas nouveaux et les cas anciens, à un moment ou pendant une période donnés.
} 
1 Sundgot-Borgen, 1994; Sundgot-Borgen \& Torstveit, 2004). De nombreuses études ont

2 rapporté une forte tendance aux désordres alimentaires, chez les compétiteurs, notamment,

3 chez les gymnastes féminines (De Bruin, Oudejan, \& Bakker, 2009 ; Rosen \& Hough, 1988).

4 Les sports esthétiques dits « à risque » prédisposeraient les athlètes au développement de

5 désordres alimentaires, en les incitant à se conformer à des formes de corps idéales, dans un

6 but d'esthétisme et de performance (Byrne \& McLean, 2002 ; Sundgot-Borgen, 1994 ;

7 Sundgot-Borgen \& Torstveit, 2004). Par contre, Augestad et Flanders (1999) ne constatent

8 pas de corrélations entre les désordres alimentaires, le niveau et le temps de pratique sportive.

La nature de la discipline, les règlements sportifs, la fréquence et la charge

d'entraînement, le commencement prématuré d'un entraînement spécifique et les normes

11

d'accomplissement imposées par la pratique sportive constitueraient un terrain propice au développement des désordres alimentaires (Afflelou, Duclos, \& Simon, 2004 ; Smolak et al., 2000 ; Sundgot-Borgen, 1994). Il faut y ajouter les pressions de l'environnement (i.e., parents, entraîneurs, équipiers ; Beals \& Manore, 2002) et la culture propre à la discipline qui perpétuent des croyances et des comportements pouvant favoriser le développement des troubles du comportement alimentaire (Sherman \& Thompson, 2009). Les habitudes alimentaires plus ou moins raisonnables développées pourraient devenir des normes, lorsque la restriction commence tôt dans la vie de l'individu (Sudi, Öttl, Payerl, Baumgartl, Tauschmann, \& Müller, 2004) et que l'individu appartient à un groupe obéissant aux mêmes lois.

\section{Facteurs psychosociaux des désordres alimentaires en sport}

Le développement de troubles du comportement alimentaire chez un individu est multifactoriel (Jacobi, Hayward, de Zwaan, Kraemer, \& Agras, 2004). Il s'agit d'une influence multiple relevant de facteurs socioculturels, interpersonnels et individuels comprenant des facteurs à la fois biologiques, héréditaires, psychologiques et 
1 comportementaux. Petrie et Greenleaf (2007) ont mis en évidence de nombreux facteurs (i.e.,

2 physiques et médicaux, psychologiques et comportementaux) qui influenceraient le

3 développement des désordres alimentaires chez le sportif. Dans cette partie, nous aborderons

4 successivement trois catégories de facteurs psychosociaux ayant un rôle prédominant dans

5 l'émergence des désordres alimentaires en sport: (a) les facteurs liés aux normes sociales, (b)

6 les facteurs liés aux influences sociales, et (c) les facteurs individuels.

\section{Facteurs liés aux normes sociales}

Normes de minceur

Dans la vie quotidienne, la pression sociale émanant des médias s'accompagne de normes relatives aux formes de corps que les individus internalisent. La recherche d'adhésion

11 à ces normes conduit parfois à l'émergence de comportements alimentaires déséquilibrés

12 (Shroff \& Thompson, 2006). Dans le contexte sportif, au-delà des normes de la vie quotidienne, les athlètes sont soumis aux contraintes du contexte d'accomplissement du sport lui-même. Ils doivent se conformer à des normes spécifiques caractérisées par un poids corporel idéal afin de s'accomplir, au travers d'une apparence physique satisfaisante et d'une excellence dans la performance (Biensecker \& Martz, 1999 ; Sundgot-Borgen 1994 ;

17 Sundgot-Borgen \& Torstveit, 2004). D'après Stice (2002), l'internalisation des normes de minceur est un facteur de risque du développement de l'insatisfaction corporelle, et par la suite, d'affects négatifs, de régimes et de symptômes de boulimie.

Les normes de minceur sont particulièrement véhiculées dans les sports à risque liés à des critères de jugements, ou dans les sports à catégories de poids ; les tenues vestimentaires de pratiques imposées pour la pratique sportive, les restrictions alimentaires conseillées, la fréquence des entraînements, les évènements traumatisants, les blessures, le surentraînement,

24 le comportement et les attentes de l'entraîneur et les règlements spécifiques aux disciplines sportives véhiculent également plus ou moins directement de telles normes (Sundgot-Borgen, 
11994 ; Smolak et al., 2000). L'athlète est souvent convaincu que la minceur est synonyme de

2 forme physique et par conséquent de performance, faisant de cette caractéristique un pré-

3 requis à la performance, entrant dans sa définition du succès sportif (Sherman \& Thompson,

4 2009).

$5 \quad$ Influence des normes groupales

Au-delà des normes de minceur imposées aux sportifs, ces derniers subissent

7 l'influence de celles du groupe de pairs. Dans le contexte de la vie quotidienne, il a été

8 démontré que ces influences sociales apparaissent dès le plus jeune âge ; par exemple, les

9 enfants se moquent souvent de leurs camarades plus enveloppés (Paxton, Schultz, Wertheim,

$10 \&$ Muir, 1999). Plus la pression sociale augmente, plus les membres du groupe deviennent

11 semblables et se conforment aux standards du groupe (Paxton et al., 1999). Dans le contexte

12 sportif, les partenaires d'entraînement et l'équipe sportive influencent les comportements, les

13 valeurs, les idées, mais aussi les images du corps et l'apparence physique des sportifs

14 (Joaquin, 2008). Carron et Hausenblas (2000) ont observé l'influence du groupe de pairs sur

15 l'alimentation et les comportements alimentaires de sportifs, à partir d'entretiens. Les

16 résultats montrent que $30 \%$ d'entre eux ressentent un effet positif de l'influence des pairs et

$1710 \%$ un effet négatif. Cet effet négatif signifie que le groupe de pairs aurait tendance à

18 favoriser l'émergence des désordres alimentaires. Un effet similaire a été observé par Ferrand,

19 Magnan, Rouveix et Filaire (2007) en natation synchronisée. Par ailleurs, Joaquin (2008)

20 évoque que le partage et l'échange concernant les habitudes alimentaires sont plus développés

21 dans les sports collectifs que dans les sports individuels. Cette différence pourrait conduire à

22 des risques plus importants chez les sportifs investis dans une pratique individuelle, en raison

23 d'un moindre soutien social. 


\section{Facteurs liés aux influences sociales}

Dans cette section, nous aborderons successivement l'influence des parents,

3 l'influence de l'entraîneur, et l'influence des pairs pesant sur les désordres alimentaires des

4 sportifs.

\section{Influence des parents}

Plusieurs études transversales et longitudinales, réalisées auprès d'enfants et

d'adolescents, ont montré que l'insatisfaction corporelle parentale perçue, le suivi de régimes,

et les préoccupations parentales concernant le poids et la minceur de l'enfant, prédisaient

significativement la mise en place de régimes, l'insatisfaction corporelle, les préoccupations à

propos du poids, la recherche de minceur ou des comportements boulimiques chez les enfants

(Keery, Eisenberg, Boutelle, Neumark-Sztainer, \& Story, 2006). Les parents, en particulier la mère, sont un relais important des pressions socioculturelles et ils constituent des sources importantes d'influence de l'image corporelle (Rodger \& Chabrol, 2009 ; Thenlen \& Cormier, 1995). Par ailleurs, les individus présentant des troubles du comportement alimentaire rencontrent souvent ou ont rencontré parallèlement des problèmes familiaux (Fairburn, 1997). Kenny et Hart (1992), sur la base de la théorie de l'attachement, ont observé que les femmes souffrant de désordres alimentaires se décrivaient comme moins solidement attachées à leurs parents que les sujets témoins.

Dans le domaine de la psychologie du sport, les recherches de ces dix dernières années ont contribué à mettre en évidence le rôle des parents sur les expériences sportives des jeunes athlètes. Les parents sont les principaux agents de socialisation des enfants lors de l'initiation sportive (Brustad, Babkes, \& Smith, 2001). Les perceptions par les jeunes des attitudes et comportements de leurs parents, dans le domaine sportif, sont associées aux perceptions de leurs propres compétences, aux affects, aux orientations motivationnelles, et aux attitudes 
Duda (2001) a examiné la valeur prédictive des climats motivationnels induits par les

2 parents sur l'estime de soi de gymnastes, leur image corporelle, leurs croyances vis-à-vis du

3 poids et leur niveau de perfectionnisme. Les résultats témoignent de la valeur prédictive de la

4 perception d'un climat familial impliquant l'ego (i.e., valorisant des critères socialement

5 référencés de la compétence) sur la croyance selon laquelle posséder un faible poids et un

6 petit corps sont des pré-requis indispensables à la réussite gymnique (Duda, 2001).

Récemment, Scoffier, Maïano et d'Arripe-Longueville (2010), dans une étude menée auprès de sportives de haut niveau investies dans des disciplines esthétiques, ont mis en évidence un caractère protecteur de la qualité de la relation avec les parents vis-à-vis des désordres alimentaires. Scoffier, Woodman et d'Arripe-Longueville (2011), ont aussi observé, dans une autre étude, que les désordres alimentaires affectaient la qualité de la relation avec les parents. Ainsi, le climat motivationnel parental et la qualité de la relation avec les parents sont deux facteurs contextuels importants en relation avec les désordres alimentaires des 14 sportifs.

\section{Influence de l'entraîneur}

L'entraîneur est un personnage spécifique à l'environnement social de l'athlète. Sundgot-Borgen (1994) a mis en évidence que l'entraîneur constitue bien souvent le personnage clef dans la vie d'un athlète, particulièrement chez les jeunes. Ainsi, aux normes sociales de minceur, s'ajoute chez les athlètes féminines en particulier, une forte pression de l'entraîneur pour tendre vers un faible poids corporel (Sundgot-Borgen, 1994). Les résultats de Scoffier, Maïano, \& d'Arripe-Longueville (2010) ont montré que la qualité de la relation avec l'entraîneur favorisait l'émergence des désordres alimentaires, par le biais de l'habileté physique perçue. Plusieurs études (De Bruin et al., 2009 ; Sundgot-Borgen, 1994 ; Ziegler,

24 Khoo, Sherr, Nelson, Larson, \& Drewnowski, 1998), ont rapporté qu'un nombre élevé 
1 correspond à une demande de la part de leur entraîneur ou à une pression de l'entraîneur. Si

2 les pratiques de régime sont renforcées par l'entraîneur, ou par les succès athlétiques, le

3 patron mis en place risque de perdurer (Biensecker \& Martz, 1999) et potentiellement de

4 mener à de sérieux désordres alimentaires. Toutefois, les entraîneurs ne peuvent pas être

5 considérés comme les seuls responsables des désordres alimentaires au travers d'un coaching

6 inapproprié ; ils peuvent seulement exacerber le problème sur un individu vulnérable

7 (Thornton, 1990).

Par ailleurs, Biensecker et Martz (1999) ont étudié comment le style de coaching était relié à l'image corporelle et aux préoccupations relatives au poids et aux régimes chez les athlètes. Deux conceptions du coaching ont été comparées : (a) dans la conception positive, le coach était concerné par la façon dont l'athlète considère sa prise de poids et la manière dont cela affecte sa vie scolaire ou universitaire - l'entraîneur était concerné par la santé physique et le bien-être psychologique de l'athlète ; (b) dans la conception négative, le coach était centré sur comment la performance de l'athlète pourrait être affectée par la prise de poids et comment les performances de l'athlète pourraient se répercuter sur le score de l'équipe l'entraîneur était intéressé par la prise de poids de l'athlète uniquement par rapport à la façon dont elle affecte les scores de l'équipe et l'esprit de compétition. Les résultats montrent qu'un style de coaching négatif est lié à de plus grandes perturbations de l'image du corps, davantage de préoccupations liées au poids et au régime, pour les garçons comme pour les filles. Par ailleurs, Duda et Kim (1997) ont mis en évidence que le climat motivationnel induit

21 par l'entraîneur influençait l'image corporelle des gymnastes et leurs croyances vis-à-vis du 22 poids. Ainsi, un climat de l'entraîneur impliquant l'égo prédisait davantage de désordres 23 alimentaires, qu'un climat motivationnel impliquant la tâche (i.e., valorisant des critères 24 autoréférencés de la compétence). 


\section{Influence des pairs}

Les pairs sont à l'origine de relations amicales et d'acceptation sociale dans le contexte sportif. L'amitié est un sentiment d'affection ou de sympathie d'une personne pour une autre, ou entre deux personnes, qui ne se fonde ni sur la parenté, ni sur l'attrait sexuel et qui se développe dans le temps (Hartup, 1983). La qualité de l'amitié se réfère globalement à la satisfaction, à la construction, à l'intimité, à la symétrie et aux substrats affectifs d'une relation dyadique (Hartup, 1996). L'acceptation sociale renvoie, quant à elle, à la « popularité » d'un individu au sein d'un groupe (Hartup, 1996).

Gerner et Wilson (2005) ont simultanément examiné les influences de l'acceptation sociale des pairs et de la qualité de l'amitié sur les désordres alimentaires et la perturbation de l'image du corps. Leurs résultats indiquent que l'acceptation sociale des pairs et de la qualité de l'amitié contribuent significativement à la prédiction de l'insatisfaction corporelle et aux restrictions alimentaires. Parallèlement, plusieurs études (e.g., Lieberman et al., 2001 ; Oliver et Thelen, 1996 ; Paxton et al., 1999) suggèrent que les amis et les pairs sont à l'origine d'une sous-culture qui, à travers les processus d'acceptation et de pression sociale et/ou d'agressivité relationnelle, influenceraient les comportements alimentaires, notamment chez les adolescentes. Ces études ont montré que les enfants intègrent, au travers de leurs expériences sociales et culturelles, qu'ils seront mieux acceptés socialement s'ils sont minces. Comme le contexte sportif, les travaux de Scoffier, Maïano \& al. (2010) ont mis en évidence, chez des sportives de haut niveau pratiquant des sports à visée esthétique, que la qualité de l'amitié en sport était un prédicteur positif de l'émergence des désordres alimentaires, par le biais de l'habileté physique perçue ; en revanche, un rôle protecteur de l'acceptation sociale des pairs envers les désordres alimentaires de ces sportives a été identifié. Cette étude confère donc un statut particulier à la qualité de l'amitié en sport. 
Les travaux les plus récents s'orientent vers l'étude d'influences sociales multiples sur

2 les désordres alimentaires. Par exemple, Kerr, Berman et De Souza (2006) ont examiné, sur la

3 base d'une approche qualitative, l'impact des parents, des sportives, des entraîneurs et des

4 juges sur les désordres alimentaires de sportives. De même, les travaux de Scoffier, Maïano et

5 al. (2010 ; Scoffier, Paquet, d'Arripe-Longueville, 2010a) illustrent cette tendance dans

6 l'explication des désordres alimentaires des sportifs.

\section{Facteurs individuels}

Les caractéristiques individuelles influencent, elles aussi, les comportements alimentaires. Elles peuvent être d'ordre psychologique (e.g., croyances, connaissances, sentiment d'auto-efficacité, goûts, et les préférences sur la nourriture), ou encore d'ordre biologique, (e.g., appétit, âge, sexe) (Story, Neumark-Sztainer, \& French, 2002). De plus, selon le National Institute of Mental Health, un certain nombre de traits de personnalité seraient symptomatiques et influenceraient le développement des désordres alimentaires.

Dans cette partie, nous aborderons, différents facteurs individuels liés à l'accomplissement du sportif et dont les relations avec les désordres alimentaires ont été étudiées : (a) l'estime de soi et ses composantes, (b) la motivation d'accomplissement, (c) le perfectionnisme ; (d)

l'autorégulation des comportements alimentaires ; (e) le locus de contrôle ; et (f) les émotions. Estime de soi et ses composantes

L'estime de soi est l'expression d'une approbation ou d'une désapprobation portée sur soi-même. Elle résulte de l'évaluation individuelle de la divergence entre l'image de soi et le soi idéal (Lawrence, 1988). Une faible estime de soi serait le facteur clé de l'obsession du corps et de la perte de poids. En effet, une faible estime de soi est souvent considérée comme un facteur de vulnérabilité vis-à-vis des troubles du comportement alimentaire (Gual , PérezGaspar, Martinez-Gonzalez, Lahortiga, De Irala-Estevez, \& Cervera-Enguix, 2002 ; Johnson et al., 2004 ; Sassoroli \& Maria Ruggiero, 2004 ; Tomori \& Rus-Makovec, 2000). Dans le 
1 contexte de la vie quotidienne, la corrélation entre faible estime de soi et insatisfaction dans la

2 perception de son poids, menant à la dépression, pourrait être un processus à double sens. La

3 perception négative de soi ferait partie intégrante de la dépression, pouvant s'exprimer par un

4 avilissement de soi (Tomori \& Rus-Makovec, 2000).

De nombreux travaux se sont intéressés aux effets de l'image du corps et ont montré

6 que, dans le contexte de la vie quotidienne, l'image négative du corps et l'insatisfaction

7 corporelle étaient généralement reliées à plus de désordres alimentaires (cf. Jacobi et al., 2004

8 pour une revue ; Shroff \& Thompson, 2006 ; Stice, 2002). La perception d'une faible

9 apparence physique pourrait augmenter le risque de désordres alimentaires, aussi bien dans la

10 population générale (Levine \& Smolak, 2006 ; Stice, 2002), que dans certains contextes

11 sportifs, tels la natation synchronisée (Ferrand et al., 2007 ; Petrie \& Greenleaf, 2007).

12 Cependant, Ferrand, Champely et Filaire (2009) et Scoffier, Maïano et al. (2010) n'ont pas observé d'effet significatif de l'apparence physique perçue sur les désordres alimentaires dans les sports esthétiques. De Bruin et al. (2009) ont, par contre, montré que les gymnastes de haut niveau comme les gymnastes investies dans une pratique régulière, avaient une image de leur corps positive. Cette relation est confirmée par les résultats de Ziegler et al. (1998) qui ont montré une relative satisfaction de leur corps chez les patineuses (voir Sherman \& Thompson, 2009 pour une revue).

Dans le contexte du sport, les perceptions du soi physique incluent non seulement

20 l'apparence physique perçue mais aussi l'habileté physique perçue (Marsh, 1990). Ces

21 dimensions seraient particulièrement mises en péril dans les sports à caractère esthétique,

22 notamment à haut niveau (e.g., Ferrand, Magnan, \& Antonini Philippe, 2005 ; Petrie \&

23 Greenleaf, 2007 ; Scoffier Maïano et al., 2010). L'habileté physique perçue a été peu étudiée

24 en lien avec les désordres alimentaires. Scoffier, Maïano et al. (2010) ont observé que cette 25 variable était un prédicteur positif des désordres alimentaires. Ainsi, la perception d'une 
1 habileté physique perçue élevée constituerait un facteur de risque des désordres alimentaires

2 chez les sportives de haut niveau pratiquant une discipline esthétique.

\section{Motivation à l'accomplissement}

Dans la littérature en psychologie sociale, les auteurs se sont intéressés à la motivation

d'accomplissement selon plusieurs conceptualisations. Initialement, le modèle (Ames \&

Ames, 1984 ; Dweck \& Legett, 1988 ; Nicholls, 1984) se basait sur une approche

bidimensionnelle des conduites d'accomplissement (buts de maîtrise vs. buts de performance). Plus récemment, plusieurs auteurs (e.g., Cury, Elliot, Da Fonséca, \& Moller, 2006 ; Elliot \& McGregor, 2001) ont proposé un renouvellement conceptuel de la théorie des buts d'accomplissement intégrant la distinction conceptuelle entre motif d'approche et motif d'évitement pour les buts de maîtrise et de performance, aboutissant au modèle $2 \times 2$ (Elliot \& McGregor, 2001).

Dans la lignée de ce que nous avons déjà exposé concernant l'influence du climat parental, Duda et collaborateurs ont mis en évidence qu'un climat de maîtrise semblait avoir une influence protectrice sur les désordres alimentaires chez les gymnastes féminines, tandis que la perception d'un climat de performance semblait avoir une influence négative sur

17 l'image du corps des gymnastes féminines, sur leurs préoccupations à propos du poids, et favoriser les désordres alimentaires en lien avec une faible estime de soi (Duda, 2001 ; Duda \& Kim, 1997).

Plus récemment, De Bruin et al., (2009), considérant le modèle bidimensionnel des buts d'accomplissement, ont observé qu'une forte orientation vers l'ego (i.e., valorisant des critères socialement référencés de définition de la compétence) était reliée à plus de comportements de régime, à une tendance au perfectionnisme plus élevée, à plus de pression des pairs par rapport au poids, et à une plus faible estime de soi. Les travaux de Chi (2004) suggèrent que les buts de performance pourraient amener à l'adoption de comportements 
1 néfastes tels la prise de drogue, d'alcool, ou les comportements de contrôle du poids. Par

2 contre, la perception d'un climat de maîtrise serait corrélée à moins de comportements de

3 régime, et moins de pression des pairs et des entraîneurs par rapport au poids. Récemment,

4 Scoffier et ses collaborateurs (Scoffier, Corrion, \& d'Arripe-Longueville, 2009 ; Scoffier,

5 Gernigon, \& d'Arripe-Longueville, en révision) ont mis en évidence que le modèle $2 \times 2$ des

6 buts d'accomplissement pouvait s'appliquer aux désordres alimentaires chez les sportifs. En

7 effet, les buts de performance-approche et les buts de maîtrise-évitement sont apparus comme

8 des prédicteurs positifs de l'émergence des désordres alimentaires et comme des prédicteurs

9 négatifs de l'autorégulation des comportements alimentaires en sport. Par contre, les buts de

10 performance-évitement et les buts de maîtrise-approche se sont avérés être des facteurs

11 protecteurs des désordres alimentaires et des prédicteurs positifs de l'autorégulation des

12 comportements alimentaires en sport.

\section{Comparaison sociale}

Il a été montré que la théorie de la comparaison sociale (Festinger, 1954) pouvait être pertinente pour comprendre les désordres alimentaires (Shaw \& Waller, 1995). La comparaison sociale désigne le processus par lequel l'individu évalue ses opinions et ses aptitudes en se référant à autrui. En effet, l'individu ne possède pas toujours de base objective (i.e., il ne peut parfois pas se référer à la « réalité physique ») pour évaluer ses opinions ou certaines de ses capacités (Festinger, 1954). La littérature en psychologie sociale et en psychologie du sport montre que la comparaison sociale est positivement reliée aux désordres alimentaires. Par exemple, Morisson et al. (2003) ont observé l'influence de la comparaison sociale dispositionnelle sur les désordres alimentaires : (a) dans un groupe clinique, la comparaison sociale était reliée à une tendance à des restrictions alimentaires ; (b) dans un groupe non clinique, la comparaison sociale était reliée aux tendances boulimiques et à l'insatisfaction corporelle. Plusieurs études (Bamford \& Halliwell, 2009 ; Shroff \& 
1 Thompson, 2006) ont confirmé une influence positive de la comparaison sociale sur

2 l'insatisfaction corporelle et les désordres alimentaires chez les étudiants et chez les

3 adolescentes. Dans l'étude de De Bruin et al. (2009), menée auprès de gymnastes féminines et

4 de danseuses, certaines sportives avaient suggéré que leurs difficultés liées à l'alimentation

5 apparaissaient quand l'entraîneur comparait leurs corps et leurs performances avec le corps et

6 les performances des autres gymnastes de leur équipe. En revanche, Scoffier et al. (2009) ont

7 montré que la comparaison sociale limitait l'émergence des désordres alimentaires en sport.

8 Des travaux supplémentaires sont nécessaires pour identifier le rôle de cette variable.

9 Perfectionnisme

10 Le perfectionnisme est un trait de personnalité pouvant avoir des conséquences

11 positives en termes de motivation pour la réussite, ou des conséquences négatives

12 caractérisées par des comportements névrotiques, obsessionnels-compulsifs (Hamachek,

13 1978). L'aspect perfectionniste lié à la présentation de soi, dans lequel le sujet évite de

14 présenter toute imperfection ou toute autre difficulté, serait aussi une dimension importante

15 dans les troubles du comportement alimentaire pour plusieurs raisons. D'une part, car

16 l'apparence physique et le poids sont des éléments centraux du concept de soi, et d'autre part,

17 car la prise de conscience de l'échec vis-à-vis du contrôle du poids afin d'arriver à un idéal de

18 minceur, pourrait rappeler d'autres échecs dans d'autres domaines (Hewitt, Flet, \& Ediger, 19 1995).

20 Dans le contexte sportif, il apparaît que le perfectionnisme négatif qui est une des

21 caractéristiques de l'athlète d'élite, pourrait expliquer l'apparition des désordres alimentaires

22 (Byrne \& Mc Lean, 2001; Garner, Rosen, \& Barry, 1998 ; Haase, Prapavessis, \& Owens,

23 2002). Par ailleurs, le perfectionnisme orienté vers soi favorise la mise en place de régimes

24 directement et par le biais de l'image du corps relative au poids chez les sportifs (Ferrand et 
1 al., 2007) et demeure relié aux désordres alimentaires dans la population générale (Castro-

2 Fornieles et al., 2007).

Capacités d'autorégulation

L'autorégulation est un concept pluridisciplinaire qui correspond à la régulation d'un système par lui-même. En psychologie sociale, l'autorégulation concerne la capacité que possède un individu à contrôler lui-même son comportement ou à réaliser une activité (Bandura, 1991). L'autorégulation des comportements se développe par un jeu d'influences réciproques de l'individu et de l'environnement, incluant le niveau des standards sociaux et moraux de l'individu (Bandura, 1991). L'autorégulation apparaît comme un puissant prédicteur des comportements de santé (AbuSabha \& Achterberg, 1997). Dans le contexte sportif, Scoffier et al. (2010a) ont montré à partir du questionnaire SREASS (Scoffier, Paquet,

12 Corrion \& d'Arripe-Longueville, 2010b, mesurant la capacité d'autorégulation des comportements alimentaires chez les sportifs, une influence négative de l'autorégulation des comportements alimentaires (en contexte d'affects négatifs, d'interactions sociales et d'absence d'anticipation des conséquences sur la performance), sur les désordres alimentaires. Ainsi, plus les sportifs ont des capacités d'autorégulation des comportements alimentaires élevées, moins ils auraient tendance à développer des désordres alimentaires (Scoffier et al., 2010a).

Locus de contrôle

Le locus de contrôle est une expectation de contrôle, définissant le fait que l'individu pense être ou non à l'origine des renforcements obtenus (Rotter, 1966). Levenson (1972) a mis en évidence qu'une structure à trois facteurs semble, à ce jour, être la plus pertinente (i.e.,

23 locus de contrôle interne, externe lié aux autrui significatifs, externe lié à la chance). Le

24 concept de locus de contrôle est un prédicteur important des comportements alimentaires 25 (AbuSabha \& Achterberg, 1997 pour une revue). 
Dans le contexte sportif, Scoffier et al. (2010a) ont montré dans une étude transversale

2 : (a) une relation positive entre le locus de contrôle interne et l'autorégulation des

3 comportements alimentaires dans un contexte d'interactions sociales ; (b) une relation

4 négative entre le locus de contrôle interne et les désordres alimentaires, par le biais de

5 l'autorégulation des comportements alimentaires en contexte d'interactions sociales, et (c)

6 une relation négative entre le locus de contrôle externe concernant l'entraîneur et l'ami en

7 sport et les désordres alimentaires par le biais, à la fois de l'autorégulation des comportements

8 alimentaires en contexte d'affects négatifs, d'interactions sociales et d'absence d'anticipation

9 des conséquences sur la performance.

Les différentes influences du locus de contrôle mises en évidence dans cette étude

ainsi que les travaux de Scoffier et al. (2009) sur les antécédents des désordres alimentaires en sport, suggèrent l'existence d'une réelle influence de l'environnement social sur les désordres alimentaires chez les sportifs.

\section{Emotions}

Dans sa méta-analyse, Stice (2002) a montré que les affects négatifs prédisaient dans la plupart des études, l'augmentation des symptômes des désordres alimentaires. Par ailleurs, Haase et al. (2002) se sont intéressés à la relation entre l'anxiété et les désordres alimentaires. L'anxiété est un trouble émotionnel qui se manifeste par un sentiment d'insécurité, une impression d'appréhension, de grande inquiétude et s'accompagnant de symptômes physiques.

L'anxiété physique et sociale associée au perfectionnisme négatif est un prédicteur des désordres alimentaires chez les athlètes d'élite (Haase et al., 2002). Par ailleurs, les scores d'anxiété dans ses différentes composantes (i.e., trait et état) sont plus élevés chez les sportives atteintes de désordres alimentaires, que chez des sportives non atteintes (Vadar, Vadar, \& Kurt, 2007). 
Par ailleurs, d'autres travaux sur la population générale suggèrent que les stratégies de

2 coping individuelles pourraient avoir une influence sur les désordres alimentaires des athlètes.

3 Le terme de coping (i.e., faire face) fait référence à l'ensemble des processus qu'un individu

4 interpose entre lui et un événement éprouvant, afin d'en maîtriser ou diminuer l'impact sur son

5 bien-être physique et psychique (Lazarus \& Launier, 1978). Plusieurs études (e.g., Bail \&

6 Lee, 2000 ; Lobera, Estébanez, Fernández, Bautista, Garrido, 2009) ont rapporté que les

7 individus adoptant des stratégies de coping de type évitement, ont un réseau de soutien social

8 insuffisant, un haut niveau de stress dans la vie, et seraient des individus plus à risque

9 concernant les désordres alimentaires.

10 Discussion générale, limites des études actuelles et perspectives de recherche

De nombreux facteurs psychosociaux d'ordres socioculturel, interpersonnel ou

individuel expliquent, en partie, l'émergence des désordres alimentaires chez les sportifs comme le montre la Figure 1. Les résultats de la littérature précédemment exposés, confirment le caractère multifactoriel de l'explication des désordres alimentaires, conformément aux données de la littérature en psychologie sociale et en psychologie du sport (e.g., Jacobi et al., 2004 ; Petrie \& Greenleaf, 2007). Cet état des lieux relatif aux recherches sur les facteurs psychosociaux des désordres alimentaires en sport a permis de mettre en évidence que certains prédicteurs des désordres alimentaires dans la population générale étaient également identifiables dans le contexte du sport de haut niveau (e.g., normes sociales de minceur, influences parentales, anxiété, perfectionnisme). spécifiques au contexte sportif qui ne sont pas observés dans le contexte de la vie quotidienne, ou qui sont appréhendés sous des appellations ou formes différentes. Par exemple, dans le contexte sportif de haut niveau, l'habileté physique perçue apparaît comme reliée positivement aux désordres alimentaires. La qualité de la relation avec l'entraîneur et avec 
1 l'ami en sport apparaissent comme des facteurs prédicteurs des désordres alimentaires par le

2 biais de l'habileté physique perçue. Dans le contexte de la vie quotidienne, le concept

3 d'habileté physique perçue n'a pas de sens ; il est supplanté par celui d'apparence physique

4 perçue. La compréhension du rôle des facteurs psychosociaux des désordres alimentaires en

5 sport nécessite donc bien des travaux prenant en compte des variables spécifiques.

L'examen des travaux évoqués dans cette revue de la littérature permet en outre

7 d'identifier un certain nombre de limites et de dégager des perspectives de recherche pour

8 tenter de mieux comprendre les désordres alimentaires en sport.

$9 \quad$ Limites des études actuelles

Plusieurs limites des études actuelles sur les désordres alimentaires en contexte sportif peuvent être identifiées. En premier lieu, la plupart des travaux réalisés reposent sur des devis corrélationnels et transversaux, et utilisent des mesures auto-rapportées. Ainsi, la portée explicative de certains facteurs psychosociaux peut-elle être discutée, et des études expérimentales intégrant des mesures comportementales devront être envisagées. En deuxième lieu, la plupart des travaux n'intègre qu'un seul temps de mesure des désordres alimentaires. Des études longitudinales, avec des mesures répétées, notamment avec des essais interventionnels randomisés, mériteraient ainsi d’être développées. Plus largement, et au regard de la littérature existante, trois catégories d'études pourraient être impulsées. Tout d'abord, il faut admettre que très peu d'études ont porté sur le rôle des facteurs socioculturels et des normes sportives sur les comportements alimentaires des athlètes. En particulier l'étude du rôle des médias (Shroff \& Thompson, 2006) et des héros sportifs (Carr, Wegand, \& Jones, 2000) sur l'internalisation des normes, les perceptions de soi et les comportements alimentaires reste inexplorée. De même, des recherches interculturelles méritent d'être encouragées. Deuxièmement, concernant le rôle de certaines variables dispositionnelles, il serait intéressant d'étudier les désordres alimentaires des 
1 sportifs dans le cadre du modèle en cinq facteurs de la personnalité (McCrae \& Costa, 1997),

2 afin d'identifier les profils d'athlètes potentiellement les plus à risque. Troisièmement, dans la

3 continuité des travaux conduits dans le cadre des théories des buts d'accomplissement, la

4 considération de variables additionnelles telles que les théories implicites de l'habileté

5 (Dweck, 1986) apporterait un éclairage complémentaire, et indispensable à la compréhension

6 des désordres alimentaires chez les individus engagés dans un contexte d'accomplissement.

Pour conclure, les facteurs psychosociaux reliés aux désordres alimentaires chez les sportives pratiquant des disciplines esthétiques sont pour certains identiques à ceux observés au sein de la population générale (e.g., influences parentales, perfectionnisme, anxiété) ce qui permet d'envisager une généralisation des travaux issus du contexte de la vie quotidienne au contexte sportif. Toutefois, certains facteurs psychosociaux reliés aux désordres alimentaires chez les athlètes paraissent spécifiques au contexte sportif (e.g., influence de l'entraîneur, influence des pairs, influence de l'habileté physique perçue). Ceci confère un caractère particulier à l'examen des facteurs psychosociaux reliés aux désordres alimentaires, notamment dans les disciplines jugées sur des critères esthétiques, et cela nécessite de poursuivre activement des investigations scientifiques dans ce champ.

L'ensemble de ces connaissances scientifiques devrait permettre, à terme, d'élaborer des stratégies de prévention des désordres alimentaires adaptées au contexte sportif, et de proposer des interventions spécifiques auprès de l'ensemble des acteurs du milieu sportif.

20 Cette démarche éducationnelle, qui pourrait être mise en œuvre auprès des sportifs, des entraîneurs voire des parents de manière concertée, devrait avoir des retombées tant dans le domaine de la formation des cadres sportifs des fédérations françaises sportives concernées, que dans celui de la préparation mentale et de la préservation de la santé des sportifs. 


\section{Références}

2

AbuSabha, R., \& Achterberg, C. (1997). Review of self-efficacy and locus of control for nutrition and health-related behavior. Journal of American Dietetic Association, 97, $1122-1132$.

Afflelou, S., Duclos, M., \& Simon, S. (2004). Quels liens entre pratiques sportives et troubles du comportement alimentaire ? Presse Medicale, 33, 1601-1605.

Ames, R., \& Ames, C. (1984). Research on motivation in education: Student motivation. New York: Academic Press.

Augestad, L. B., \& Flanders. W. D. (2002). Eating disorder behavior in physically active Norwegian women. Scandinavian Journal of Medicine and Science in Sports, 12, 248255.

Bail, K., \& Lee, C. (2000). Relationships between psychological stress, coping and disordered eating: A review. Psychology and Health, 14, 1007-1036

Bamford, B., \& Halliwell, E. (2009). Investigating the role of attachment in social comparison theories of eating disorders within a non-clinical female population. European Eating Disorders Review: The Journal of the Eating Disorders Association.

Bandura, A. (1991). Social cognitive theory of moral thought and action. In W. M. Kurtines \& J. L. Gewirtz (Eds.), Handbook of moral behavior and development: Theory, research and applications: Vol. 1. (pp. 45-103). Hillsdale, NJ: Lawrence Erlbaum Associates, Inc.

Beals K. A., \& Manore, M. M. (2000). Behavioral, psychological and physical characteristics of female athletes with subclinical eating disorders. International Journal of Sport Nutrition Exercise Metabolism, 10, 128-143. 
1 Biesecker, A., \& Martz, D. (1999). Impact of coaching style on vulnerability for eating disorder: An analog study. Eating Disorders: Journal of Treatment and Prevention, 7 , $235-244$.

Brustad, R., Babkes, M. L., \& Smith, A. L. (2001). Youth in sport: Psychological considerations. In R. N. Singer, H. A. Hausenblas, \& C. M. Janelle (Eds.) Handbook of Sport Psychology (pp. 604-633). New York: Wiley.

Byrne, S, \& McLean, N. (2001). Eating disorders in athletes: A review of the literature. Journal of Science Medical in Sport, 4, 145-169.

Carr, S., Weigand, D. \& Jones, J. (2000). The relative influence of parents, peers, and sporting heroes on goal orientations of children and adolescents in sport. Journal of Sport Pedagogy, 6, 34-55.

Carron, A. V., Hausenblas, H. A. (2000). Group influences on eating and dieting behaviors in male and female varsity athletes. Journal of Sport Behavior, 23, 33-41.

Castro-Fornieles, J., Gual, P., Lahortiga, F., Gila, A., Casulà, V., \& Fuhrmann, C. (2007). Self-oriented perfectionism in eating disorders. International Journal of Eating Disorders, 40, 562-568.

Chi, L. (2004). Achievement goal theory. In T. Morris, J. Summers (Eds.), Sport psychology: Theory, applications and issues ( $2^{\text {nd }}$ Ed.) (pp.152-174). Australia: John Wiley Son.

Cury, F., Elliot, A., Da Fonséca, D., \& Moller, A. (2006). The social-cognitive model of achievement motivation and the $2 \mathrm{X} 2$ achievement goal framework. Journal of Personality and Social Psychology, 90, 666-679.

De Bruin, K., Bakker, F. C., \& Oudejans, R. D. (2009). Achievement goal theory and disordered eating: Relationships of disordered eating with goal orientations and motivational climate in female gymnasts and dancers. Psychology of Sport Exercise, 10, $172-79$. 
1 Duda, J. (2001). La théorie des buts d'accomplissement et la santé dans le domaine physique. In F. Cury, \& P. Sarrazin, (Eds.), Théories de la motivation et pratiques sportives : état des recherches, (pp. 255-276). Paris : PUF.

Duda, J. L., \& Kim, M. (1997). Perceptions of the motivational climate, psychological characteristics, and attitudes toward eating among young female gymnasts. Journal of Sport and Exercise Psychology, 19, S48, (NASPSPA abstracts).

Dweck, C. S., \& Leggett, E. L. (1988). A social-cognitive approach to motivation and personality. Psychological Review, 95, 256-273.

Elliot, A. J., \& McGregor, H. A. (2001). A 2x2 achievement goal framework. Journal of Personality and Social Psychology, 80, 501-519.

Fairburn, C. G. (1997). Interpersonal psychotherapy for bulimia nervosa: In Handbook of Treatment for Eating Disorders ( $2^{\text {nd }}$ Ed.). D. M. Garner P. E. Garfinkel, (Eds.), (pp. 278-294). New York: Guilford.

Fairburn, C. G., \& Harrison, P. J. (2003). Eating disorders. Lancet, 361, 407-416.

Ferrand, C., Champely, S., \& Filaire, E. (2009). The role of body esteem in predicting disordered eating symptoms: A comparison of French aesthetic athletes and non-athletic females. Psychology of Sport and Exercise, 10, 373-380.

Ferrand, C., Magnan, C. \& Antonini Philippe R. (2005). Body-esteem, body mass index and risk for disordered eating among adolescents in synchronized swimming. Perceptual and Motor Skills, 101, 877-884

Ferrand, C., Magnan, C., Rouveix, M., \& Filaire, E. (2007). Disordered eating, perfectionism and body-esteem of elite synchronized swimmers. European Journal of Sport Science, 7, 223-230.

Festinger, L. (1954). A theory of social comparison processes. Human Relations, 7, 117-40. 
1 Garner, D. M., Olmstead, M. P., \& Polivy. J. (1983). Development and validation of a multidimensional eating disorder inventory for anorexia nervosa and bulimia. International Journal of Eating Disorders, 2, 15-34.

Gerner, B., \& Wilson, P. (2005). The relationship between friendship factors and adolescent girls' body image concern, body dissatisfaction, and restrained eating. International Journal of Eating Disorders, 37, 313-320.

Gual, P., Pérez-Gaspar, M., Martinez-Gonzalez, M. A., Lahortiga, F., De Irala-Estevez, J., \& Cervera-Enguix, S. (2002). Self-esteem, personality, and eating disorders: baseline assessment of a prospective population-based cohort. International Journal of Eating Disorders, 31, 261-273.

Haase, A. M., Prapavessis, H., \& Owens, R. G., (2002). Perfectionism, social physical anxiety and disordered eating: a comparison of male and female elite athletes. Psychology of Sport \& Exercise, 3, 209-222.

Hamachek, D. E., (1978). Psychodynamics of normal and neurotic perfectionism. Psychology, $15,27-33$.

Hartup, W. W. (1983). Peer relations. In E. M. Hetherington (Ed.), Handbook of child psychology, Vol. 4 (pp. 103-196). New York: Wiley.

Hartup, W. W. (1996). The company they keep: Friendships and their developmental significance. Child Development, 67, 1-13.

Hewitt P. L., Flett G. L., \& Ediger E. (1995). Perfectionism traits and perfectionistic selfpresentation in eating disorder attitudes, characteristics, and symptoms. International Journal of Eating Disorders, 18, 317-326.

Hobart, J. A. \& Smucker, D. R. (2000). The female athlete triad. American Family Physician, $61,3357-3367$. 
1 Jacobi, C., Hayward, C., de Zwaan, M., Kraemer H. C., \& Agras, S. (2004). Coming to terms with risk factors for eating disorders : application of risk terminology and suggestions for a general taxonomy. Psychological Bulletin, 13, 19-65.

Joaquin, D. (2008). Eating disorders in athletes. England, J. Wiley Sons, Inc.

Johnson, C., Crosby, R., Engel, S., Mitchell, J., Powers, P. S., Wittrock, D., \& Wonderlich, S. (2004). Gender, ethnicity, self-esteem and disordered eating among college athletes. Eating Behaviors, 5, 147-156.

Keery, H., Eisenberg, M. E., Boutelle, K. N., Neumark-Sztainer, D., \& Story, M. (2006). Relationships between maternal and adolescent weight-related behaviors and concerns: The role of perception. Journal of Psychosomatic Research, 51, 105-111.

Kenny, M., \& Hart, K. (1992). Relationship between parental attachment and eating disorders in an inpatient and a college sample. Journal of Counseling Psychology, 39, 521-526.

Kerr, G., Berman, E., \& De Souza, M.J. (2006). Disordered eating patterns in elite, female gymnasts. Journal of Applied Sport Psychology, 18, 28-43.

Lawrence, D. (1988), Enhancing Self-esteem in the classroom. London, Paul Chapman Publishing.

Lazarus, R.S., \& Launier, R. (1978) Stress-related transactions between person and environment. In L.A. Pervin \& M. Lewis (Eds), Perspectives in Interactional Psychology. (pp.287-327). New York: Plenum

Levenson, H. (1972). Distinctions within the concept of internal-external control: development of a new scale. Proceedings of the 80th annual convention of the American Psychological Association, 261-262.

Levine, M. P., \& Smolak, L. (1996). Media as a context for the development of disordered eating. In L. Smolak, M. P. Levine, R. \& Striegel-Moore (Eds.), The developmental 

psychopathology of eating disorders: Implications for research, prevention, and treatment (pp. 235-257). Mahwah, NJ: Lawrence Erlbaum.

Lieberman, M., Gauvin, L., Bukowski, W. M., \& White, D. R., (2001). Interpersonal influence and disturbed eating behaviors in adolescent girls: The role of peer modelling, social reinforcement, and body-related teasing. Eating Behaviors, 2, 216-236.

Lobera, I. J., Estébanez, S., Fernández, M. J. S., Bautista, E. A., \& Garrido, O. (2009). Coping strategies in eating disorders. European Eating Disorders Review: The Journal of the Eating Disorders Association, 17, 220-226.

Marsh, H. (1990). A multidimensional, hierarchical model of self-concept: Theoretical and empirical justification. Educational Psychology Review, 2, 77-172.

McCrae, R.R., \& Costa, P.T. (1997) Personality trait structure as a human universal. American Psychologist, 52, 509-516.

Morisson, T., Wallers, G., Meyer, C., Burditt, E., Wrigth, F., Babbs, M., \& Gilbert, N. (2003). Social comparison in the eating disorders. The Journal of Nervous and Mental Disease, $191,553-555$.

Nicholls, J. G. (1984). Achievement motivation: Conceptions of ability, subjective experience, task choice, and performance. Psychological Review, 91, 328-346.

Oliver, K, \& Thelen, M. (1996). Children's perceptions of peer influence on eating concerns. Behavior Therapy, 27, 25-39.

Paxton, S. J., Schutz, H. K., Wertheim, E. H., \& Muir, S. L. (1999. Friendship clique and peer influences on body image concerns, dietary restraint, extreme weight-loss behaviors, and binge-eating in adolescents girls. Journal of Abnormal Psychology, 108, 255-266.

Petrie, T. A., \& Greenleaf, C. A. (2007). Eating disorders in sport: From theory to research to intervention. In G. Tenenbaum (Ed.), Handbook of Sport Psychology, 3rd Edition (pp. 352-378). New Jersey: J. Wiley Sons, Inc. 
1 Rodgers, R., \& Chabrol, H. (2009). Parental attitudes, body image disturbance and disordered eating amongst adolescents and young adults: a review. European Eating Disorders Review: The Journal of the Eating Disorders Association, 17, 137-151.

Rosen, L., \& Hough, D. (1988). Pathogenic weight control behaviors in female college gymnasts. Physician Sports Medicine, 16, 141-146.

Rotter, J. B. (1966). Generalized expectancies for internal versus external control of reinforcement. Psychological Monograph, 80, 1-28.

Sassaroli, S., \& Maria Ruggiero, G. (2005). The role of stress in the association between selfesteem, perfectionism, worry, and eating disorders. International Journal of Eating Disorders, 37, 135-141.

Scoffier, S., Corrion, K., \& Arripe-Longueville, F. (d') (2009). Effect of achievement goals and social comparison on elite athletes' eating disorders. Communication orale au Congrès International de l'Association for Applied Sport Psychology.

Scoffier, S., Gernigon, C., \& Arripe-Longueville, F. (d') (en révision). Effects of Achievement goals on self-regulation of eating attitudes among elite female athletes: an experimental study. Psychology of Sport \& Exercise.

Scoffier, S., Maïano, C., \& Arripe-Longueville, F. (d') (2010). The effects of social relationships and acceptance on disturbed eating attitudes in elite adolescent female athletes: the mediating role of physical self-perceptions. International Journal of Eating Disorders.

Scoffier, S., Paquet, Y., \& Arripe-Longueville, F. (d') (2010a). Effect of locus of control on disordered eating in athletes: The mediational role of self-regulation of eating attitudes. Eating Behaviors, 11, 164-169. 
1 Scoffier, S., Paquet, Y., Corrion, K, \& Arripe-Longueville, F. (d') (2010b). French development and validation of the self-regulatory eating attitude in sports scale (SREASS). Scandinavian Journal of Medicine Science in Sport, 20, 696-705.

Scoffier, S., Woodman, T., \& Arripe-Longueville, F. (d') (2011). Psychosocial Consequences of Disordered Eating Attitudes in Elite Female Figure Skaters. European Eating Disorders Review, 18, 1-8.

Shaw, J., \& Waller, G. (1995). The media's impact on body image: Implications for prevention and treatment. Eating Disorders, 3, 115-123.

Sherman, R. T., \& Thompson, R. A., (2009). Body image and eating disturbance in athletes: Competing to win or to be thin? In J. J. Reels\& K. A. Beals (Eds.), The Hidden Faces of Eating Disorders and Body Image (pp. 9-38). Reston, VA: AAHPERD.

Shroff, H., \& Thompson, J. K. (2006). The tripartite influence model of body image and eating disturbance: A replication with adolescent girls. Body Image: An International Journal of Research, 3, 17-23.

Smolak, L., Murnen, S. K., \& Ruble, A. E. (2000). Female athletes and eating problems: A meta-analysis. International Journal of Eating Disorders, 27, 371-380.

Stice, E. (2002). Risk and maintenance factors for eating pathology: A meta-analytic review. Psychological Bulletin, 128, 825-848.

Story,M., Neumark-Sztainer, D., \& French, S. (2002). Individual and environmental influences on adolescent eating behaviors. Journal of the American Dietetic Association, 102, 40-51.

Sudi, K., Öttl, K., Payerl, D., Baumgartl, P., Tauschmann, K., \& Müller, W. (2004). Anorexia athletica. Nutrition, 20, 657-661.

Sundgot-Borgen, J. (1994). Risk and trigger factors for the development of eating disorders in female elite athletes. Medicine Science in Sports Exercise, 26, 414-419. 
1 Sundgot-Borgen, J., \& Torstveit, M. K., (2004). Prevalence of eating disorders in elite athletes is higher than in the general population. Clinical Journal of Sport Medicine, 14, 25-32.

4 Thelen, M. H., \& Cormier, J. F. (1995). Desire to be thinner and weight control among children and their parents. Behavior Therapist, 26, 85-99.

6 Thornton, J. S. (1990). Feast or famine: Eating disorders in athletes. Physician Sports Medecine, 18, 116-122.

Tomori, M., \& Rus-Makovec, M. (2000). Eating behavior, depression, and self-esteem in high school student. Society for Adolescent Medicine, 26, 361-367.

Vardar, E., Vardar, S.A., \& Kurt, C., (2007). Anxiety of young female athletes with disordered eating behaviors. Eating Behaviors, 8, 143-147. Eating Disorders, 24, 421-427. 
Figure1. Modèle Etiologique Hypothétique des Facteurs Psychosociaux Influençant les Désordres Alimentaires en Sport.

Notes. $n s$ : relation non-significative ; BMA : Buts de Maîtrise-Approche ; BPE : Buts de Performance-Evitement ; BME : Buts de MaîtriseEvitement ; BPA : Buts de Performance-Approche. 